\title{
Intelligent Products: a survey
}

\author{
Gerben G. Meyer \\ Department of Business 8 ICT, Faculty of Economics and Business, University of Groningen, P.O. Box 800, 9700 AV \\ Groningen, The Netherlands \\ Kary Främling \\ Department of Computer Science and Engineering, Helsinki University of Technology, P.O. Box 5500, FIN-02015 TKK \\ HUT, Finland
}

Jan Holmström

Department of Industrial Management and Engineering, Helsinki University of Technology, P.O. Box 5500, FIN-02015 TKK HUT, Finland

\begin{abstract}
This paper presents an overview of the field of Intelligent Products. As Intelligent Products have many facets, this paper is mainly focused on the concept behind Intelligent Products, the technical foundations, and the achievable practical goals of Intelligent Products. A novel classification of Intelligent Products is introduced, which distinguishes between three orthogonal dimensions. Furthermore, the technical foundations in the areas of automatic identification and embedded processing, distributed information storage and processing, and agent-based systems are discussed, as well as the achievable practical goals in the contexts of manufacturing, supply chains, asset management, and product life cycle management.
\end{abstract}

Key words: intelligent products, product lifecycle, software agents, supply chains, RFID

\section{Introduction}

This paper gives an overview of the recently emerged field of Intelligent Products, by analyzing and proposing definitions of what they are and by performing a survey of how they have been or can be implemented and used in different application areas. In this context, Intelligent Products and concepts like Smart Products can be used interchangeably. However, Intelligent Products are not a synonym for concepts like Ubiquitous and Pervasive Computing, Ambient Intelligence etc. that tend to focus on how human users interact with their environment. The Internet of Things [33] concept could be a better fit, but it tends to be focused rather on connectivity and information exchange than on the "intelligence" of the products. Intelligent Products are not always invisible and unnoticeable, they are rather reactive actors that are capable of autonomously adapting to changes in their environment. Even though this is still largely a vision for the future, this survey will provide a picture of the current status and how it can evolve towards this vision in different application areas.

Intelligent Products have many facets. In this survey, the concept, technical implementation, and achievable practical goals will be reviewed. Based on that review, a classification of Intelligent Products will be introduced, which distinguishes between three orthogonal (albeit not independent from each other) issues: what is the intelligence of the product, what is the location of intelligence, and whether the product consists of a single entity or if it is an aggregation or composition of several entities. The reason for introducing such a three-dimensional classification is that previously proposed classifications seemed to be under-developed either in the lower or the upper range of "intelligence" and did not necessarily take into account e.g. how the

Email addresses: g.g.meyer@rug.nl (Gerben G. Meyer), kary.framling@hut.fi (Kary Främling), jan.holmstrom@hut.fi (Jan Holmström) 
embedded processing capabilities affect the implementation of such Intelligent Products. Previous classifications also tend to focus only on limited parts of a product's lifecycle, e.g. manufacturing or maintenance only, rather than taking into account the entire lifecycle. Analyzing different approaches to Intelligent Products using the proposed three-dimensional classification makes it easier to identify what their limitations are, and which are the main aspects that need further development.

Furthermore, the technical foundations of Intelligent Products will be discussed in detail in this survey. These foundations can mainly be found in the areas of automatic identification and embedded processing, distributed information storage and processing, and agent-based systems. In order to keep the survey reasonably limited, some other relevant domains such as technologies for fault detection and remote maintenance are largely omitted from this survey. For these domains, there already exists dedicated journals and other dissemination channels. Also, challenges related to privacy, security, trust etc. are not discussed in detail here, but such aspects in the context of Intelligent Products are discussed in detail in e.g. [35, 50, 51, 71. Some other challenges for implementing Intelligent Products, such as the cost and the availability of skilled personnel are pointed out in e.g. [23] and [45].

Regarding how, when and why to implement Intelligent Products, the achievable goals for the Intelligent Product concept will be presented as a starting point for developing practical business cases in individual companies. It is important to note that solutions developed for one purpose can, if appropriately designed, be employed for other purposes as well. Means-ends propositions [68 for Intelligent Products in specific contexts will be discussed, such as manufacturing, supply chain, and asset management, as well as across contexts, i.e. for product life cycle management.

\subsection{Background}

In the early days, factories were often powered by one central steam engine. As mentioned in [10, the electric engine was meant to replace the steam engine. Just as there was one steam engine that would power an entire factory, the electric motor was also initially a single device installed at a central location in the factory, with belts running to the remote areas of the factory. The electric motor improved to the point where a single motor with belts could be replaced by motors built into each device. Now you could place the instruments wherever it made sense to put them, and the motor became an invisible part of the instrument.

According to Norman [60, computers and computer networks should be thought of as infrastructure. It should be quiet, invisible and unobtrusive, instead of being too visible and too demanding. He therefore envisages a change occurring from one centrally located infrastructure (the personal computer), to a set of rather small, widely distributed devices. These devices will not even be thought of as computers or telecommunication devices. Instead, these devices will be seen as a natural part of our daily activities and the tools that we use [10. He argues that the proper way to achieve this is through the user-centered, humane technology of appliances, where the technology of the computer disappears behind the scenes into task-specific devices that maintain all the power without the difficulties [60. Similar views about computing had earlier been proposed mainly under the name ubiquitous computing (see e.g. [80, 81]), that is also sometimes called pervasive computing or ambient intelligence depending on the context.

Gershenfeld shares this vision, as he calls invisibility the missing goal in computing [34]. According to him, we can bring technology so close to people that it can finally disappear. Furthermore, he emphasizes that the barrier between digital information and our physical world should be removed. The real challenge in this is to figure out how to create systems with many components that can work together and adapt to changes in the physical world. This vision has sometimes been called the Internet of Things, which is also adopted e.g. in [13, 33, 36. However, in many contexts such as Supply Chain Management, the Internet of Things concept tends to be focused on product identification technologies, information storage and information exchange rather than on the "intelligence" of the products.

It seems like Intelligent Products were first discussed in an after sales and service context in 1988 by Ives and Vitale [37. The first examples of Intelligent Products in the after sale context were computers running programs that tracked the configuration and performance, and could request for service and maintenance. The benefits in efficiency of service and reliability of operation could be substantial and was the basis for successful start-ups and new lines of business for established companies.

Only later did the idea of integrating intelligence and control into the product spread to manufacturing [55] and supply chain control [47. In these application domains, new auto identification (Auto-ID) technologies, such as Radio Frequency Identification (RFID) have made the tracking and tracing of products 
throughout the entire supply chain possible. When product individuals in a logistic/production setting are not only given a traceable individuality, but also the associated content (e.g. delivery terms, contract terms, exceptions, etc.), and also decision power is delegated to them, we enter the realm of Intelligent Products. Such Intelligent Products will have the means to communicate between themselves and also with logistic service providers. Intelligent Products link the Auto-ID technology to the agent paradigm and Artificial Intelligence. Agent technology has already been considered as an important approach for developing industrial distributed systems (e.g. intelligent manufacturing systems) [39, 40, 66.

Intelligent Products can also play an essential role in product lifecycle management by their capability of collecting usage information and reacting on it proactively, e.g. estimating needs for maintenance or repair [62. By using sensor technologies like thermal, acoustic, visual, infrared, magnetic seismic or radar sensors the conditions of products can be continuously monitored. The access to information on how products have been used could significantly improve the way that products are recycled when they arrive to their end-of-life. Sensor technologies can also contribute to improvements in manufacturing nodes and to the logistics of the entire supply chain, by giving real-time status information (e.g. identification, location and other conditions) of the products.

What is common to such tracking and tracing in the supply chain and to product lifecycle management is that information needs to be represented at the item level and communicated between different organizations. From an information system perspective, a shipment is indeed just a "product" with a relatively short lifecycle, where the actual products that were included in the shipment may have a much longer lifecycle. However, currently used information systems typically focus on managing batches and accounts using centralized databases, hence representing item-level information and communicating it between organizations can be a challenge for them, in case of mass-customization of products. Therefore, there is increasing interest in the development of Auto-ID technologies and Intelligent Products which is being reflected in on-going work, current project proposals and future research areas.

\subsection{Paper outline}

After this introduction, section 2 will analyze different proposals for defining Intelligent Products. Furthermore, a classification method for such products that can be used as a tool for classifying the different implementation approaches will be presented. Section 3 gives an overview of the enabling technologies of Intelligent Products. Section 4 will analyze how Intelligent Products can be implemented and used in different application domains, followed by conclusions and future trends in section 5

\section{What are Intelligent Products}

This section will start with presenting existing definitions of Intelligent Products found in the literature. All these definitions focus on certain aspects of Intelligent Products and on certain application areas or parts of the product lifecycle. Afterwards, based on the existing definitions, a classification of Intelligent Products will be proposed, which tries to cover all aspects of Intelligent Products while taking into account the whole product lifecycle.

\subsection{Definitions of Intelligent Products}

From the existing definitions of Intelligent Products, the complementary notions of McFarlane et al., Kärkkäinen et al. and Ventä will be discussed next.

\subsubsection{McFarlane et al.}

McFarlane et al. define an Intelligent Product as a physical and information-based representation of a product [55]. Figure 1 shows an example of such a product. In this figure, the jar of spaghetti sauce is the physical product, the information-based representation of the product is stored in the database, and the intelligence is provided by the decision making agent. The connection between the physical product and the information-based representation is made using a tag and a reader, as will be further discussed in section 3.1 . According to McFarlane et al., an Intelligent Product has the following properties:

1. Possesses a unique identification

2. Is capable of communicating effectively with its environment 


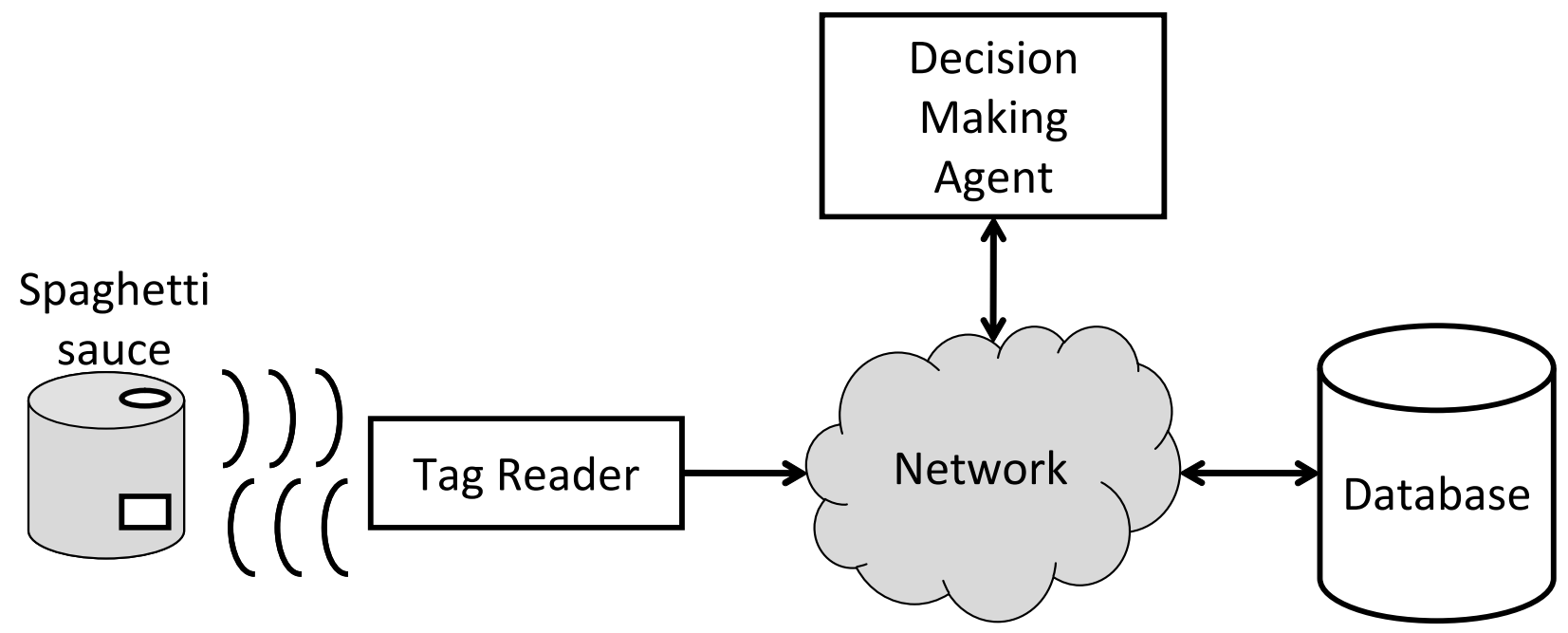

Figure 1: Intelligent jar of spaghetti sauce (from [82])

3. Can retain or store data about itself

4. Deploys a language to display its features, production requirements, etc

5. Is capable of participating in or making decisions relevant to its own destiny

Based on this definition, Wong et al. 82 have defined a two-level classification of intelligence. When the Intelligent Product only covers points 1 to 3 , it is information oriented, and is called a product with level 1 product intelligence. A product with level 2 product intelligence covers all points, and is called decision oriented. Even though this Intelligent Product classification is quite generic concerning the level of intelligence of an Intelligent Product, it is based on a separation between the actual product and its information-based counterpart (as seen in figure 1). Therefore, it is mainly intended for describing the use of RFID technology in for example manufacturing and supply chain purposes, without covering for instance products with embedded processing and communication capabilities.

\subsubsection{Kärkkäinen et al.}

The fundamental idea behind an Intelligent Product according to Kärkkäinen et al. [47] is the inside-out control of the supply chain deliverables and of products during their lifecycle. In other words, the product individuals in the supply chain themselves are in control of where they are going, and how they should be handled. To move to inside-out control of products, the products should possess the following properties:

1. Globally unique identification code

2. Links to information sources about the product across organizational borders, either included in the identification code itself or accessible by some look-up mechanism

3. Can communicate what needs to be done with them to information systems and users when needed (even pro-actively)

In this definition, the classification goes from no intelligence (unique identification only) towards decisionoriented products when covering the last property, in a similar way to the classification by McFarlane et al. Despite a slightly bigger consideration for embedded processing capabilities and the whole product lifecycle, this classification is still mainly focused on the use of RFID technology, similar to McFarlane et al's classification.

\subsubsection{Ventä}

Another definition of Intelligent Products is given by Ventä in [79]. Ventä refers by intelligence to products and systems that:

1. Continuously monitor their status and environment

2. React and adapt to environmental and operational conditions 
3. Maintain optimal performance in variable circumstances, also in exception cases

4. Actively communicate with the user, environment or with other products and systems

This definition is clearly focused on decision-oriented products, thereby extending point five of the first definition and point three of the second definition. However, this definition is more focused on products with sufficient embedded computing power for communicating directly with other information systems. The main application area is the running and maintenance of products in use, with little or no consideration of manufacturing and supply chain management. This is a noteworthy difference with the first two definitions, as the first two mainly deal with products that only possess an identification such as a barcode or an RFID tag, thereby requiring external information storage and communication facilities.

\subsection{Classification of Intelligent Products}

All three definitions focus on certain aspects of Intelligent Products and the product lifecycle, and thereby cover only a part of the total field of Intelligent Products. Therefore, we need a more comprehensive classification of Intelligent Products that covers all the aspects of the field. This classification can be used for analyzing different information architectures according to what kind of Intelligent Products and what parts of the product lifecycle they are suited for. A classification based on three orthogonal dimensions will be presented in the remainder of this section.

\subsubsection{Level of intelligence}

The degree of intelligence of an Intelligent Product can vary from "dumb" products to pro-active entities. This is the main focus of the definitions and classifications of McFarlane et al. and Kärkkäinen et al. Based on these definitions, the level of Intelligence of Intelligent Products can be divided into three categories:

- Information handling. An Intelligent Product should at least be able to manage its own information, given by sensors, RFID-readers and other techniques. Without this capability, it can hardly be called intelligent. When the Intelligent Product is only capable of information handling, it is not in control of its own life, as full control of the product is external or outside the product.

- Problem notification. A more Intelligent Product is a product which can notify its owner, when there is a problem. Such a problem could for example be that it has fallen, the temperature is too high, etc. Still the product is not in control of its own life, but it's able to report when there are problems with its status.

- Decision making. The most Intelligent Product is the product which can completely manage its own life, and is able to make all decisions relevant to this by itself, without any external intervention. In this case, the product has full control over itself, and there is no external or outside control of the product. This has been called inside-out control of products in [47].

\subsubsection{Location of intelligence}

When each object has its own intelligence, it does not necessary mean that the intelligence is located at the object. Two extremes can be identified:

- Intelligence through network. The intelligence of the product is completely outside the physical product, at a different location. For example, there is a server where a dedicated agent for the product is running [26]. The definitions of McFarlane et al. and Kärkkäinen et al. are mainly focused on this approach. The product only contains a device that is used as an interface to the intelligence. In the research field for smart devices, such devices are often called small SD (Smart Device) [16, 17. Platforms in which the intelligence of the product is executed entirely on other hosts are sometimes called portal platforms 63 .

- Intelligence at object. All the intelligence, whether this is only information handling, or advanced decision making, takes place at the physical product itself. The definition of Ventä is mainly focused on this approach. The object has the needed computational power, storing capacities and network connectivity. In the research field for smart devices, such devices are often called big SD [16, 17]. Platforms in which the intelligence of the products is executed entirely on the devices are sometimes called embedded platforms [63]. 
There can also be intermediate solutions, these platforms are sometimes called surrogate platforms [63]. In section 3 the techniques underlying these differences will be further elaborated.

\subsubsection{Aggregation level of intelligence}

A third dimension of Intelligent Products is one which is lacking in the definitions and classifications discussed in the first part of this section. However, we consider the aggregation level of the intelligence also as an important dimension, as many products are composed from parts, which can also be products in itself. For example, a car is an assembly of components that are manufactured by different organizations and that may by themselves be composed of other parts. In the case of modern cars or other products with sufficient information processing and communication capabilities, a lot of decision-making can be embedded into the product itself. However, some parts of the product may have only an identifier, while other parts may have their own embedded information processing capabilities. In order to make it possible to access information in a uniform way from all levels, at least the communication interface should be similar for all components of the product, as proposed for instance in [29]. For analyzing this dimension, we make the following separation:

- Intelligent item. The object only manages information, notifications and/or decisions about itself. If it contains any components, they can not be distinguished as individual objects.

- Intelligent container. The intelligent container not only manages information, notifications and/or decisions about itself, it is also aware of the components that it is made of and may act as a proxy device for them. If the intelligent container is disassembled or parts are removed or replaced, the parts may be able to continue as intelligent items or containers by themselves. For instance, an engine may be removed from a vehicle, be re-furbished and then start a new life in another vehicle, possibly together with new or re-furbished components (alternator, clutch, etc.). Another example from the domain of supply chain management is an intelligent shelve, which can notify its owner when a specific product is out of stock.

\subsubsection{Classification model}

Together, these dimensions lead to a three-dimensional classification model for Intelligent Products, which covers all the main aspects of the field. This classification model is shown in figure 2, In section 4 the classification model will be used to classify the Intelligent Products in the discussed applications.

\section{Technologies enabling Intelligent Products}

This section analyzes the technologies behind Intelligent Products from three main points of view. Section 3.1 starts with the identification, sensing and information processing technologies that can be embedded in the product itself, ranging from barcodes to embedded computers with sensors, network connectivity etc. Section 3.2 discusses how the intelligence can be "outsourced" from the product itself to other storage and processing devices such as back-end systems. Three different approaches for how this could be implemented are studied with the objective to illustrate their possibilities and challenges. Finally, in section 3.3 different agent-based platforms are discussed that have been proposed for addressing the challenges of local versus distributed information storage and processing.

\subsection{Automatic Identification and embedded processing}

Already in the early 1970's, the first optical scanning systems using barcodes were installed to provide production line part tracking, as well as to satisfy the needs of companies for inventory and order fulfillment visibility down the supply-chain, and also to provide production line part tracking. [23, 72]. The adoption of the UPC standard in 1973 caused a sudden increase of barcode scanning, leading to a widespread use of laser scanning to track items ranging from convenience store purchases to overnight delivery packages. However, the disadvantages of these systems are a slow information flow, since bar codes are a line-of-sight technology that requires manual scanning and allows only one item to be read at a time. Consequently, bar codes are only read at a few control points in the supply-chain. Thus arose the need for a new auto identification (Auto-ID) technology such as RFID, which overcomes these limitations.

RFID is a wireless data collection technology that relies on tags, consisting of silicon memory chips equipped with radio antennas, which can be attached to objects to transmit streams of data about them. 


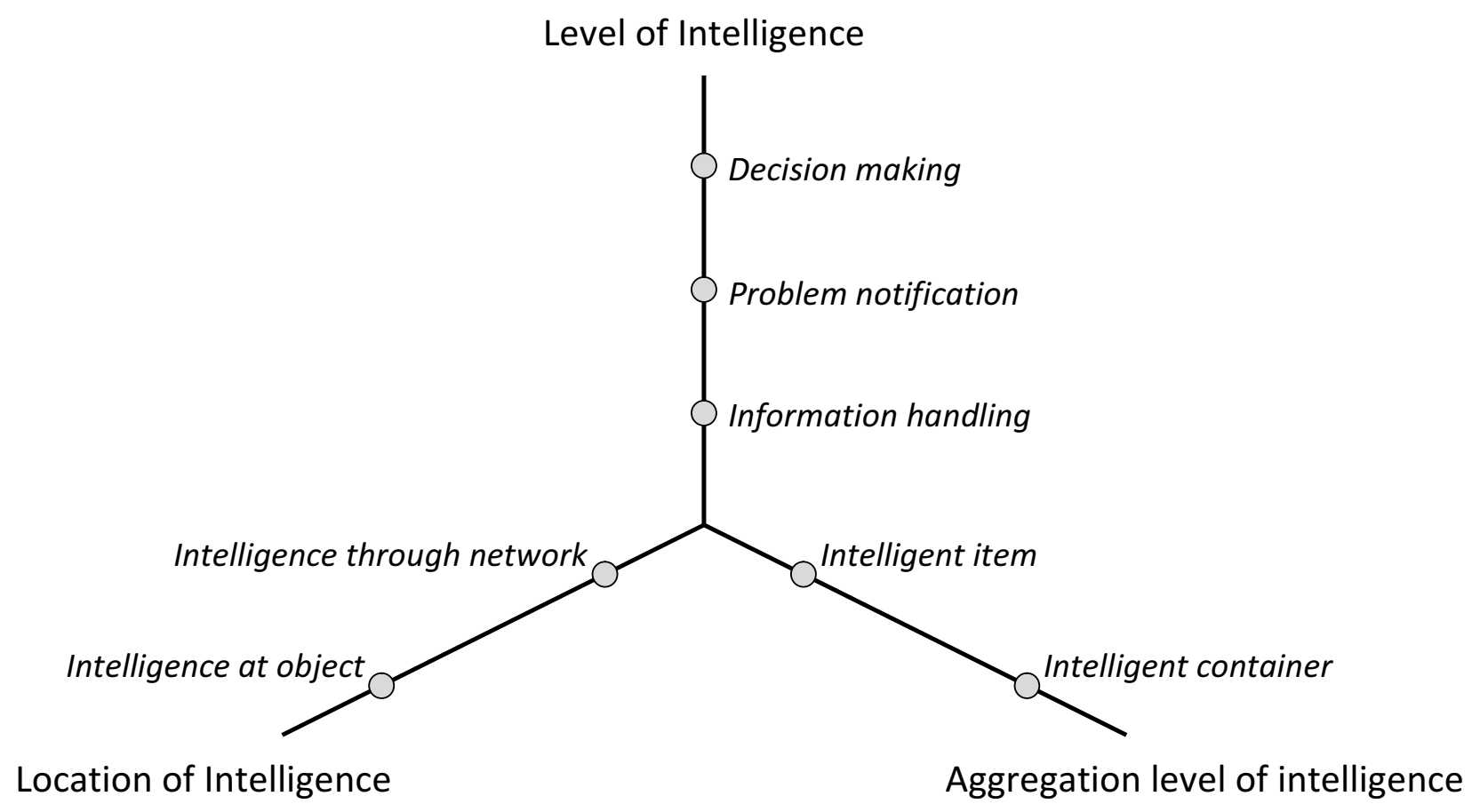

Figure 2: Classification model of Intelligent Products

RFID tags, unlike bar codes, do not require a reader to come in direct contact with an item, nor do they require a line of sight between reader and tag. RFID tags are also more suitable than barcodes for identifying unique individuals, instead of only identifying them on the product type level. Furthermore, multiple tags can be read simultaneously. RFID tags are more difficult to counterfeit than bar codes because their manufacturerassigned serial number is hard-wired into the chip. The data on the chip can also be protected from reading and writing in various ways, including encryption in more expensive tags. RFID tags can be categorized based on different aspects [70]:

- Memory: read only, read/write, or a combination. The read/write capability of a tag can be used for reading and recording data on the chip as it moves through the system. Tags with read only memory normally only store a unique identifier code.

- Active or passive. Active tags are powered with an internal battery that gives them a longer reading range and the possiblity to include sensors and actuators. Passive tags draw power from the readers, making them lighter, smaller, and cheaper to produce.

- Frequency bands. Low-frequency tags are used in applications where the range is generally less than 25 centimeters, while high-frequency tags are used in areas of less than a meter. Ultra-high frequency tags have a longer reading range, currently up to about eight meters in optimal conditions. However, these distances tend to increase constantly as new technologies are developed.

As mentioned before, Auto-ID technologies, such as barcode, RFID, smart card, and biometric systems, are commonly used to identify products or delivery units. In addition to automatic identification, Auto-ID technologies often also include localization and sensor technologies. Localization techniques are often combined with automatic identification, as the location information is useless without the identity of the located entity [73]. The location of a product can be approximated using various techniques [72, 73]: monitoring by wireless and cellular access points, alteration and angulation of radio frequency or ultrasonic signals (e.g. Global Positioning System), scene analysis (e.g. image recognition), laser trackers (e.g. Coordinate-Measuring Machines), as well as micro-sensors and micro-electromechanical systems (MEMS), Inertial Navigation Systems (MEMS INS) and MEMS Optical Identification and Communication Systems (MOICS). A detailed discussion of these techniques is outside the scope of this paper. Another frequently applied technique is updating the 
location status of the product at the moment its barcode or RFID-tag is scanned, when the physical location of the scanner is known 36 .

Typically, barcodes and passive RFID chips only have the capacity of storing information. Especially when automatic identification technologies are combined with sensor technologies, such as thermal, acoustic, visual, infrared, magnetic seismic or radar sensors, processing of this information locally at the product can be beneficial. For this purpose, Gellersen et al. 32] look at how to integrate sensors in mobile devices, in such a way that the context of the device can improve user interaction and support new types of applications. In this way, for instance mobile phones and PDA's can be used to support products when augmented with embedded computing.

Furthermore, there is an increase in interest on applying MEMS for creating smart devices (e.g. [31, 72]). Because of the small size of these sensors and chips, these technologies are well applicable on products. In the Smart-Its project [69], experiments are already conducted on different scenarios for attaching small-scale embedded devices (Smart-Its) to everyday artifacts, to augment them with sensing, perception, computation, and communication. In this project, "Smart-Its" is regarded as an enabling technology for building and testing ubiquitous computing scenarios, and therefore they will use them to study emerging functionality and collective context-awareness of information artifacts. Siegemund and Flörkemeier discuss several possible scenarios for Smart-Its in [67]. One possible scenario for the use of Smart Its is smart product monitoring. The smart product monitoring scenario is an example of a pervasive computing scenario where the interaction is initiated by a smart object. An example is presented where an egg carton represents an arbitrary object that is in store in e.g. a warehouse. The object is augmented in such a way that it detects whenever it is dropped or not stored within the appropriate temperature range. Whenever such an exception occurs, it triggers an alarm by informing the appropriate contact person via an SMS. Another scenario mentioned by Siegemund and Flörkemeier is the smart medicine cabinet, which was designed to support mobile and young patients with chronic diseases. It is supposed to improve the drug compliance of these patients by reminding them to take their medicine. The smart medicine cabinet also knows about its content so that the user can query it remotely to check which medication he/she has currently available. Other features include out-of-date detection and alarms for potential product recalls. Unfortunately, designers apparently did not consider Smart-Its very appealing [53].

Embedded processing is mainly related to the "Intelligence at object" approach. In situations where an "Intelligence through network" approach is needed, it becomes necessary to take into consideration how product information can be managed in a distributed way that may involve the product itself, end-users, manufacturers, other supply chain members etc. This is the subject of the next section.

\subsection{Distributed information storage and processing}

The vision of Intelligent Products is to seamlessly connect the products in the physical world with their representation in information systems e.g. through a product agent as proposed in [26]. Intelligent Products would make it possible to avoid media breaks between the real word and the digital world. Thereby, data about the current and past context of objects from the physical world can be retrieved and updated when needed. As pointed out in Section 2 , the basic building blocks for implementing "intelligence through network" is that products are identified by globally unique identifiers that either encode links to information sources directly or that can be used as look-up keys in some kind of network infrastructure. The main three currently known approaches are shortly analyzed here. A deeper technical analysis and comparison can be found in [28.

\subsubsection{EPC Global}

A tracking and tracing system for products throughout the supply chain was developed by the MIT AutoID Center [65, which later has been split into EPCglobal Inc. 24], and Auto-ID Labs [6. In the EPCglobal approach (as shown in figure 3a), every product is tagged with an Electronic Product Code (EPC). The EPC is a numbering scheme that can provide unique identification for physical objects, assemblies and systems. An Object Naming Service (ONS) tells computer systems where to locate information on the Internet about any object that carries an EPC. Because of security and performance issues, EPCglobal has started defining an alternative look-up infrastructure called the "discovery services". The application layer events (ALE) and EPC information service (EPCIS) published by EPCglobal provide standardized communication interfaces for communicating product-related information. 


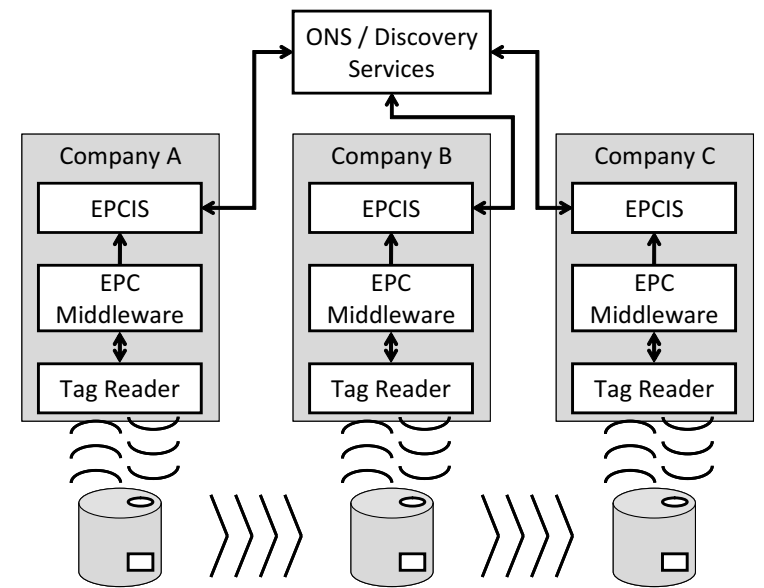

(a) EPC Global based

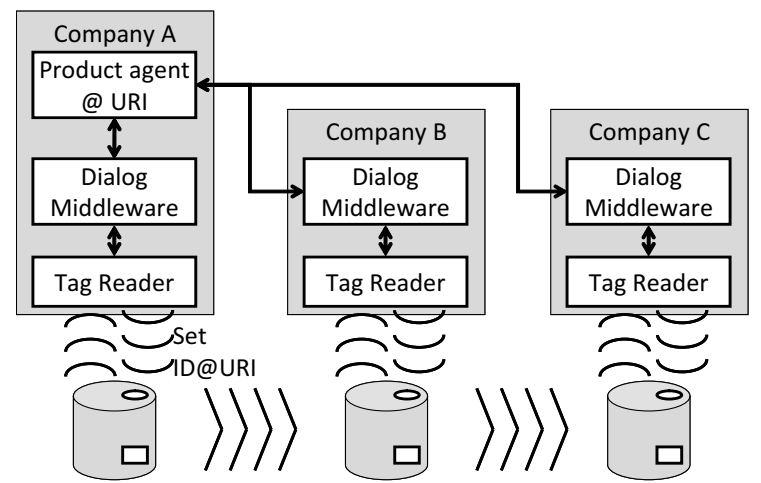

(b) ID@URI based

Figure 3: Tracking system for products moving through a supply chain

The main advantages of the EPCglobal approach are the strong industrial support and standards supported by organizations such as GS1. The main weak points are related to the fact that the proposed information architecture and standards remain focused on supply chain management applications using passive RFID tags. Supporting other Auto-ID technologies (especially high-end ones with embedded information processing and communication capabilities) may be challenging. The current lack of item-level look-up between product identifiers and related information sources is also a weakness.

\subsubsection{ID@URI}

At Helsinki University of Technology, a product identification and information linking concept labeled ID@URI [36] was proposed. With ID@URI, each product carries the ID of the product, as well as the URI (Uniform Resource Identifier) where the agent of this product can be found. Thus, ID@URI links the physical objects directly with their product agent that is implemented as an Internet-enabled service. The URI is typically the URL where the product agent is located, which could for instance be the address of a manufacturer's server computer. Because the address of the product agent is directly embedded in the identifier, the existing domain name service (DNS) infrastructure is sufficient. The ID can also be chosen freely as long as it is unique in the context of the URI, therefore existing serial numbers or numbering standards (including EPC) can be used.

An information system called the Dialog platform [22, 26, that uses ID@URI was initially developed for tracking products through a supply chain. The Dialog platform contains two software components, as can be seen in figure $3 \mathrm{~b}$. The first component is the product agent that is managing the product information. The second component is used e.g. for updating the location of shipments passing at checkpoints or for querying or updating product information in general. The checkpoint can handle barcode, RFID-tags or any identification technology capable of storing at least an ID and a URI. Extensions for handling e.g. composite products and for propagating information updates have been presented in [27, 30]. Work on the ID@URI concept is continued in the TraSer project [76].

The initial goal of the Dialog platform was to develop Intelligent Products that could respond to the challenges found in international project deliveries [47. There, the fundamental challenges come from the customized nature of project deliveries, the great number of individual deliveries to the project, the large number of suppliers, and the fact that deliveries to the project site are time-critical. In the proposed inside-out control of project deliveries, such an Intelligent Product could ask for itself when it is in need of assembling or transportation. Furthermore, it could have a more active role in after-sales, and should be able to manage its own life-cycle [47.

The main advantage of ID@URI is its simplicity and that it can be used without new standards or infrastructure. However, no communication interface standards have been proposed for the Dialog platform, which is therefore currently on a proof-of-concept level after successful industrial pilots [48, 49]. This is 
largely because the ID@URI concept and the Dialog platform can be implemented using existing or evolving standards, such as the messaging interfaces and data models developed in the PROMISE project [62].

\subsubsection{WWAI}

World Wide Article Information [83] is an application level protocol for distributed article information developed by the Trackway company [75]. WWAI uses a product identifier that combines existing GS1 identifiers for the organization, product type and an item-level serial number. This product identifier is then used as a search key for retrieving available information sources that own information about the product. Both the retrieval of information sources and the information exchange use principles of peer-to-peer networking. WWAI enables companies to share real-time product information, regardless of the Auto-ID method used, over the Internet. The WWAI protocol enables distribution of the information on the computers of the companies that have participated in the manufacturing, assembling or transporting of the product. Every participant has control of its own product information and decides whether the information is public of private. Distribution of information makes WWAI networks scalable and able to grow as the number of information provider nodes and products grow.

The main advantage of WWAI comes from its distributed nature of handling product information lookup and access, which makes it relatively fault-tolerant. WWAI also contains functionalities for managing composite products and other relations between products, as well as event propagation between organizations. The main challenge for WWAI is that it is currently not standardized and that it does not have a big installedbase that would enable it to become de-facto standard.

\subsection{Agent-based platforms}

Agents are a useful paradigm to implement Intelligent Products, as the concept of an agent is close to the concept of an Intelligent Product. An intelligent agent is defined as "a computer system, situated in some environment, that is capable of flexible and autonomous action in order to meet its design objectives" [39]. A multi-agent system is a federation of software agents interacting in a shared environment, that cooperate and coordinate their actions given their own goals and plans. For this purpose, agents typically have four properties. The first property is autonomy. This means that agents operate without the direct intervention of humans or others, and have some kind of control over their actions and internal state. The second property is social ability, where agents interact with other agents via some kind of agent-communication language. The third one is reactivity where agents perceive their environment and respond in a timely fashion to changes that occur. The fourth property is pro-activeness. Here agents do not simply act in response to their environment; they are able to exhibit a goal-directed behavior by taking the initiative.

There are several reasons why the use of an agent-based platform for Intelligent Products is beneficial. Firstly, when there is a high number of products, we will have to reduce the number of products needing explicit control from the user. This can be achieved by making the products autonomous. In this way, Intelligent Products with knowledge and reasoning capabilities can do most of the repetitive tasks in an automated way. Secondly, Intelligent Products should be able to detect and react to changes in the environment. Agents can pro-actively assist the product and try to achieve goals given the change of the environment. Agents can also help in discovering information about the environment by communicating with agents of other products. It is therefore clear that intelligent agents have characteristics which are desirable for Intelligent Products. Of course, an application for Intelligent Products can be created without the use of agents, but by using agents, one can take advantage of the methodologies and solutions provided by the multi-agent paradigm [17.

By using intelligent agents for implementing an application for Intelligent Products, each product can have its own intelligent agent. This does not necessary mean that the agent is located at the product. As discussed in section 2, two extremes can be defined. These extremes are also shown in figure 4a and 4b. Also some intermediate solutions exists, which are called surrogate platforms. Next, several platforms in those categories will be discussed. This discussion is partially based on the overview of [17.

\subsubsection{Portal platforms}

This kind of platforms mainly relate to the "intelligence through network" approach. Most standard agent platforms can be used as portal platforms, as in that case the agents are not embedded on the products, but run on "normal" desktop systems or servers. However, there are some specific platforms designed for 


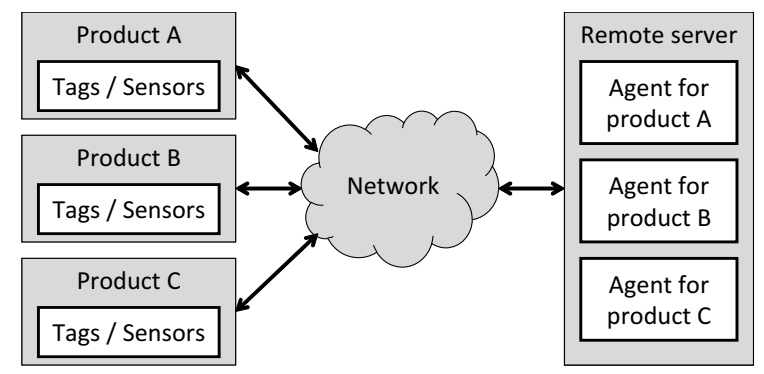

(a) Portal platform

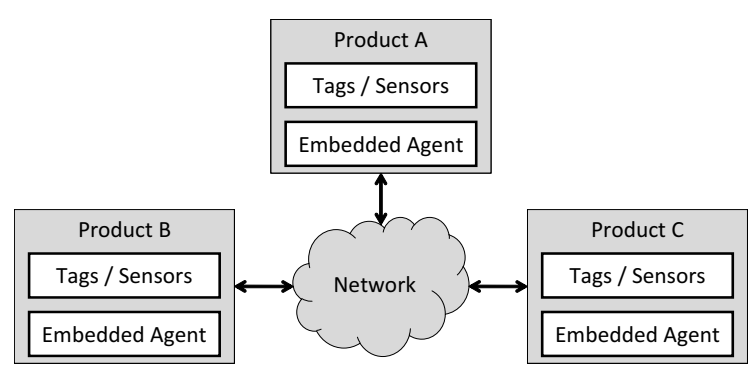

(b) Embedded platform

Figure 4: Agent-based platforms

this purpose, like the MobiAgent system. The MobiAgent [54] system architecture consists of three main components: mobile wireless devices (which the products should be equipped with in case of Intelligent Products), an agent gateway, and the network resources. The agent gateway is the location where the actual agents are executed. The mobile devices can download an interface of an agent through which an agent can be configured. The agent will perform its task and will later report the results to the mobile device via the same mechanism.

\subsubsection{Embedded platforms}

This kind of platforms mainly relate to the "intelligence at object" approach. Several platforms have been developed to support agents embedded on mobile devices, in order to enable ubiquitous multi-agent systems. These platforms are mainly based on Java, in order to work seamlessly on any Java-enabled devices with sufficient resources, like mobile phones, PDA's, and in the future even smaller devices, which can be attached to the products. The Lightweight Extensible Agent Platform (LEAP) [9] is probably the most well known agent platform for small devices. Since version 3.0, LEAP is an add-on of the Java Agent DEvelopment Framework (JADE) platform [38]. The LEAP platform can be used as both a surrogate and as an embedded platform. Other examples of embedded platforms are the 3APL-M Platform [1, 46], the MicroFIPA-OS platform [56], and the Grasshopper platform [8].

\subsubsection{Surrogate platforms}

An example of a surrogate platform is the KSACI platform [2]. This platform is an extension of the SACI (Simple Agent Communication Infrastructure) platform [64], in order to enable agents embedded in devices to exchange information and knowledge with other embedded agents or with agents located in desktop computers. Each (K)SACI agent has a mailbox to exchange messages with the other agents. The architecture contains one special agent, called the facilitator, offering white- and yellow-pages services of the system. The white-pages can be used by agents to locate other agents in the network, as the yellow-pages can be used to find agents which offer a specific service. This platform is a surrogate platform, as the facilitator agent is always running on a server. Furthermore, agents embedded on devices cannot pass messages directly to other agents, but instead they have to communicate with an intermediate HTTP server running a SACI proxy, which will deliver the messages to the appropriate receiver. The solution makes the embedded agents lighter, which makes them easier to embed on small devices.

\section{Goals of Intelligent Products}

This section outlines a number of achievable practical goals for the application of Intelligent Products. A means-ends proposition [68] is a semi-formal description of a goal that can be achieved by applying a solution in different contexts. In formulating these propositions, the goals of an application of Intelligent Products are explicated, particular solution requirements are identified, and the circumstances where the goals can be achieved are discussed. Several authors in design science and related disciplines emphasize the need for such propositions when searching for new solutions and applications. Different terms used for the means-ends proposition include: base case [44, the technical norm [58, and the technological rule [61, 78]. 


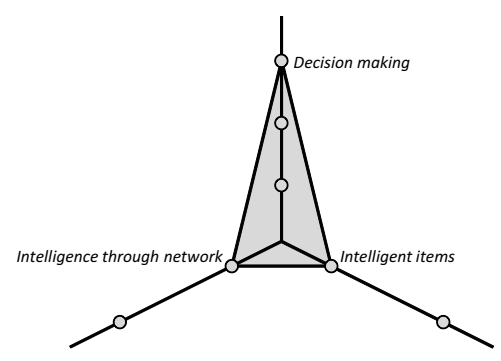

(a) West

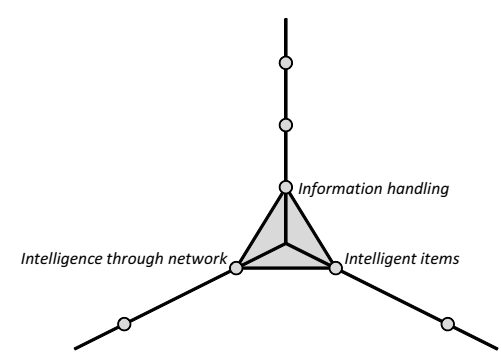

(b) QSC Audio Products

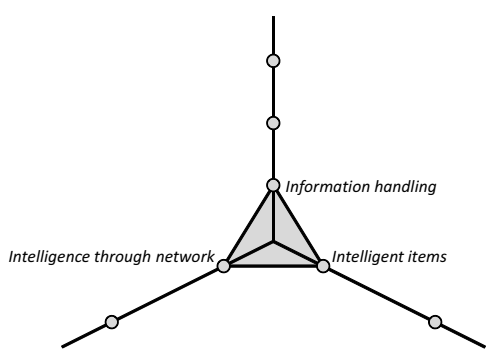

(c) SMED

Figure 5: Classification of Intelligent Products in manufacturing applications

The goals for Intelligent Products are context dependent and are reviewed for manufacturing, supply chains, asset management and product lifecycle management.

\subsection{Manufacturing}

Currently, the manufacturing industry is moving more and more from a supplier-driven to a customerdriven market. Due to the growing industrial capacity, customers are provided with a greater choice, and competition between suppliers is increased. As a result, companies must shorten product life cycles, reduce time-to-market, increase product variety and instantly satisfy demand, while maintaining quality and reducing investment costs. This is a great challenge to the manufacturing process itself; it must be more flexible and robust as well as demonstrate enhanced scalability [14. Therefore, the ends for introducing the Intelligent Product concept in manufacturing are to improve production planning and control, to enable customized products and to make change-over between product variants more effective. The classification of the Intelligent Products in the discussed applications of this section can be found in figure 5 .

\subsubsection{Production planning and control}

A first goal for companies to introduce Intelligent Products in manufacturing is to improve their current operation in terms of production planning and control. Frequent changes of production plans caused by engineering changes as well as production disturbances, such as machinery breakdown or the late or uncertain arrival of component parts, can lead to instabilities in production and production scheduling, with a 'ripple effect' on other firm functional boundaries. In [52, Lee and Kim give an overview of how multi-agent systems are used for achieving local and global objectives in production planning and control. Further, they give an overview on multi-agent systems research applied in dynamic scheduling and shop floor job assignment, as well as how to solve process planning and scheduling integration problems.

One of the first Intelligent Products application in manufacturing is the self-organizing manufacturing control system of Bussmann, which was evaluated at DaimlerChrysler [15. In the control system (called West), developed for the DaimlerChrysler concept of a modular and flexible manufacturing system, agents are assigned to both work pieces and to machines. The agent of a single workpiece negotiates with the agents of the machines about which one of the machines will process the workpiece next. The workpiece auctions off its current due operations and invites machines to bid. If a workpiece awards a specific machine, then an operation performed by this machine on the workpiece will be the next goal of the workpiece. A workpiece will continue to auction off operations and award machines until it reaches its desired state. Simulations have shown that the West mechanism is extremely robust against disturbances of machines, as well as failures of control units. According to Bussmann and Schild, its performance is nearly optimal [15, mainly due to the dynamic task allocation, with late commitment.

\subsubsection{Customized products}

A second goal for companies that have introduced Intelligent Products in manufacturing is a logical next step from the first goal. When Intelligent Products manage or assist in the production planning and control, they can be applied to control the manufacture of customized products, i.e. producing efficiently products that vary from instance to instance. An example is QSC Audio Products [25], an early user, that 


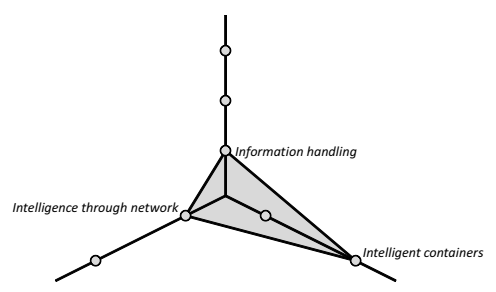

(a) Wal-Mart

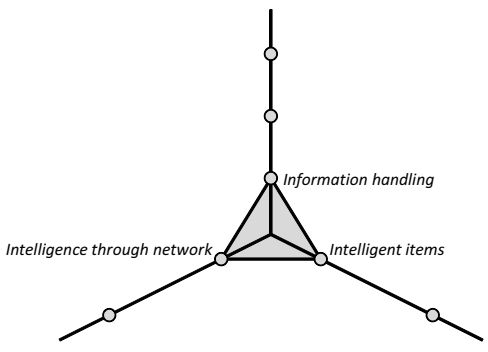

(b) Skanska

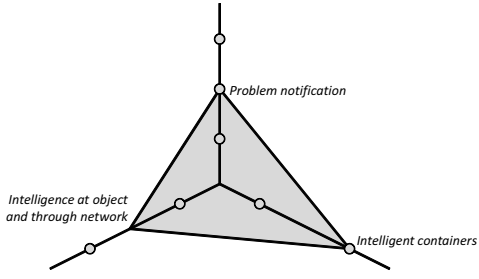

(c) ParcelCall

Figure 6: Classification of Intelligent Products in supply chain applications

was able to move from a build-to-stock operation to a build-to-order operation by introducing a solution based on RFID technology to track and control how work-in-process (WIP) moves through the facility. The application enables the company to manufacture customized products more efficiently because it can optimize the routing of work, and direct materials to where they are needed during assembly.

\subsubsection{Change-overs}

Another objective for Intelligent Product applications in manufacturing is to improve set-ups and changeover management. By developing Intelligent Product applications for containers of materials needed for different variants of standard products it becomes possible to speed-up and reduce errors in set-ups and change-overs. [77 provides a nice illustration on how setup times can be reduced by tagging, tracking, and controlling materials and tools. In trying to apply the concept of single-minute-exchange-of-dies (SMED), that was originally developed in the sheet metal fabrication context in the printed circuit board (PCB) assembly context, it was found that success required developing a simple Intelligent Product application. Material and tool tracking was needed to help operators locate and prepare the thousands of unique parts and feeders used in the circuit board assembly. Introducing SMED in a PCB fabrication context required a tracking and tracing solution that relied on the innovative use of wireless terminals, identification technology, and relational databases.

\subsection{Supply chains}

As for a few years ago, RFID technology has been successfully used in some portions of the supply chain [3, 12]. In this same supply chain context, the ends served by Intelligent Product applications are: more efficient goods issue and receipt, re-routing of deliveries in-transit, and improved security (e.g. anti-theft). The classification of the Intelligent Products in the discussed applications of this section can be found in figure 6 .

\subsubsection{Sending goods}

An end for the application of Intelligent Products in the supply chain is to improve the efficiency of sending and receiving goods. A track and trace application of British retailer Marks \& Spencer's 42 reduces the lead time for distributing the frozen food from the distribution centers to the stores. The implemented solution tracks frozen food on RFID tagged and recycled transport assets. A similar, and more widely known example is Wal-Mart. Wal-Mart has mandated its suppliers to apply RFID tagging onto pallets and boxes so that Wal-Mart can speed up receiving of goods and reduce the incidence of stock-outs in the shop [5]. A majority of Wal-Mart's suppliers have answered to the mandate by adopting a "slap-and-ship" practice to product tagging [19]. In "slap-and-ship", the suppliers apply the RFID tagging to the units just before shipping or even by a third party before the delivery to Wal-Mart. By introducing the Intelligent Product concept, for example by linking handling directions to the container, products that are out of stock in the shop can be prioritized both when dispatched and when goods are received in the store.

\subsubsection{Re-routing of deliveries}

Another goal that also is based on the Intelligent Product concept is the re-routing of products and shipments in transit. This proposition is important for example in the delivery of components for complex 


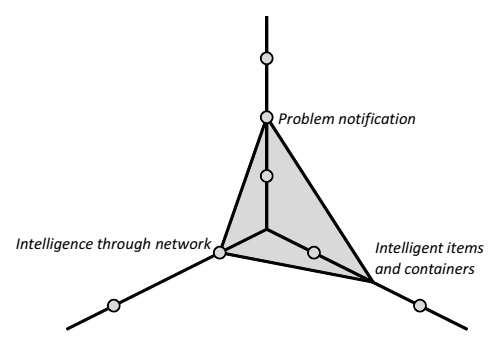

(a) U-Library

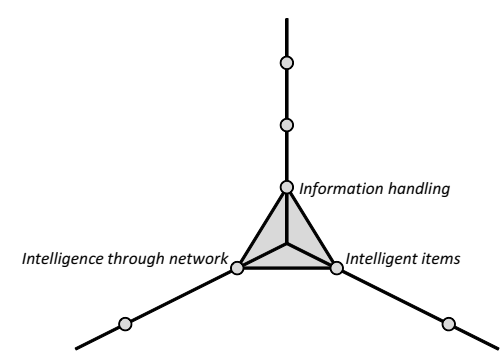

(b) ToolWatch

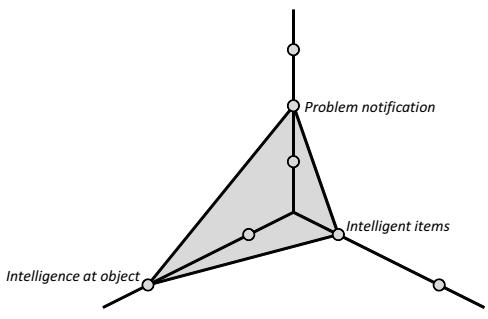

(c) Smart-It

Figure 7: Classification of Intelligent Products in asset management applications

systems where delivery to the customer site is time critical [47]. Each component delivery can be associated to an application that specifies what needs to be done with it, and this information can be used to reroute or change handling instructions to different supply chain members while the delivery is in transit. An example is the pilot by Skanska [59], a construction engineering and construction company that in a pilot started to control major pre-cast reinforced concrete components that were tagged with RFID. The component is linked to a tracking system and depending on the progress of different building sites interchangeable components can be rerouted to where they are needed the most.

\subsubsection{Security}

A third basic purpose of introducing the Intelligent Products concept in the supply chain is to improve the security of the supply chain. By maintaining the identity of the product or shipment it is possible to pinpoint where thefts occur and/or to verify the authenticity of the item and reduce the risk of forgery. An example of developing an application of Intelligent Product that improves the security of the supply chain is from the European IST project ParcelCall [21]. In the ParcelCall solution the intelligence is linked to the transport unit. Each transport unit has a mobile logistic server that keeps track of the goods within that unit. In advance of the loading process the transport unit receives over a communications network a list of contents from a goods tracking server. While the items are loaded the server associated with the transport unit checks whether the item belongs to the list of items to be loaded or not. If an item is loaded that is not in the list of contents, the transport unit sends notice to goods tracking. When the loading procedure is finished the transport unit server also notifies that the loading procedure has been completed.

\subsection{Asset management}

Asset management is the context where it is currently most straightforward to develop practically relevant means-ends propositions at least regarding RFID technology. Expensive assets such as tools and equipment often need to be used by many parties, and their continued use require the services of different service providers. By introducing intelligence to the assets it becomes easier to share assets and also to service them. The ends for introducing Intelligent Product concepts are to improve the efficiency of asset usage and also to make service and maintenance of the assets more effective. The classification of the Intelligent Products in the discussed applications of this section can be found in figure 7

\subsubsection{Efficiency}

U-Library [57] illustrates how the introduction of intelligence makes it easier to share an asset. In the u-library (ubiquitous library), each book is equipped with an RFID tag, and linked to an intelligent agent managing it. Furthermore, every shelf, browsing table, etc. is equipped with RFID readers and writers, and also linked to an intelligent agent managing it. By tracking the books using the RFID tags, it becomes possible to monitor how books and other library materials are used and how patrons move and use books inside of the library. More copies of items that are continuously used can be procured, while copies of items that are not needed can be sold-off or moved to another location. Therefore, by applying Intelligent Products in this way, the utilization can in theory be improved at the same time as the investment in assets is reduced. Another example of applying Intelligent Products in asset management is from industry. Bowen Engineering 
used a tool tracking solution called ToolWatch that combines both identification and tracking [74]. This resulted in an improved utilization of assets, as well as a big reduction in tool investments.

\subsubsection{Service and maintenance}

An example that well illustrates the benefit of the Intelligent Product concept to improve service and maintenance is from health care. A patient in a hospital or a care service needs different types of maintenance and service depending on her condition. Attaching a Smart-It device to a mobile medicine cabinet can greatly facilitate the health care of young patients with chronic diseases. Smart-It is a small-scale computing device that can be attached to different everyday objects to provide them with sensing, computing and communication capabilities [67, 69]. The Intelligent Product application makes it possible for the care giver to track medicine use and drug compliance of the patients, and to remind the patients to take their medicine. The intelligent mobile medicine cabinet also keeps account of its content so that the patient's doctor or pharmacist can query it remotely to check which medication he/she has currently available, and whether it is in need of replenishment. Other useful functions that can be easily included are out-of-date detection and support for product recalls.

\subsection{Product lifecycle management}

According to Kiritsis [45, the focus today in the business environment, is on innovation: products that differentiate themselves from others while also being affordable, reliable and early to market. Total management of the product lifecycle is critical to innovatively meet customer needs throughout the entire life cycle without driving up costs, sacrificing quality, or delaying product delivery. The ability of industry to provide such holistic products and supporting services is currently limited by the information gap in the product's life cycle. The development of ubiquitous computing (in the form of Product Embedded Information Devices (PEID)) is expected to progress rapidly and largely used for advanced Product Lifecycle Management and real-time data-monitoring throughout the Product Supply Chain. The basic idea behind PEIDs is to store information about an individual product on the product itself, by applying technologies as discussed in section 3.1

Practical business cases of the lifecycle management proposition have been addressed in the PROMISE project [62. An information architecture has been defined that makes it possible to collect information from any lifecycle phase and to use it in the same or any other phase of the product lifecycle. The information architecture defines communication interfaces based on Web Services technology for communication over the Internet and a UPnP technology for short-range communication especially with mobile PEIDs that have intermittent network connectivity. Any software that implements either one of these interfaces can be a PROMISE information provider. A system object model developed in PROMISE [18, proposes a universal way for storing and interpreting lifecycle-related data. The developed model would extend current ISO STEP and PLCS (Product Life Cycle Support) standards especially for managing information gathered during the use of products, including consumer products such as cars, refrigerators and other potentially "intelligent" products.

\subsubsection{Improved product design, manufacturing and customization}

Applications of Intelligent Products that have been developed for a specific context can in certain situations be extended across many stages in the product lifecycle. For example, an application that was initially developed for controlling customization in manufacturing can perhaps also be used for improved handling in the supply chain, and to support efficient maintenance in asset management. Car manufacturers Ford, BMW and Vauxhall already use RFID-tags to improve product quality, to store information about the car during its whole life cycle, and to enable accurate customization of customer orders [7, 11, 41, 84]. An additional purpose of this type of lifecycle application of the Intelligent Product concept is better coordination between product development, service operations and sales. For example, using the same Intelligent Product platform the manufacturer collects information to improve its product development, the efficiency of its service operations, and to identify more accurately sales and marketing opportunities.

\subsubsection{Maintenance}

The proposed information architecture can be used to manage and control information on product individuals in the manufacturing and delivery process, as well as after the product has been sold [4. The PEIDs 
handle information on the product individual level (and possibly also on part and sub-assembly level), instead of on the product type level, as typically is the case with current product data management (PDM) systems. The PEIDs can store data about the products locally (i.e. at the product itself) which enables the distribution of analysis and control tasks to the product itself. In addition to local analysis and alerts to the user/owner, the distributed information architecture supports alerts to the manufacturer or service provider when certain thresholds are violated or abnormal conditions are detected. In this way, failure of a product can be better prevented, and the product can many times be replaced before breaking down.

\subsubsection{End-of-life management}

Information about usage conditions can also be used when the product is at its end-of-life for determining how to handle the product and its parts. When combining usage information with design and manufacturing information, it is possible to determine the presence of valuable material or hazardous substances and create a list of parts that have to be removed or special treatments that are required. In some industries, there are also databases where the currently estimated market value of spare parts is available. When combining the usage information with a value estimate, it becomes possible to calculate a residual value of each part and make an even more fine-grained decision of what is the most appropriate action to take for each part. Such possibilities could enable new recycling and refurbishing business opportunities as in the automotive industry [43] or for heavy machinery as identified by Caterpillar [20].

\section{Conclusions and Future Trends}

In this survey, the concept, technical implementation, and achievable practical ends of Intelligent Products have been reviewed. As discussed in the introduction of the paper, the Intelligent Product concept is not very well-defined because it combines many disciplines and could be used in many ways. The need for a new classification of Intelligent Products that was identified in Section 2 illustrates that we are dealing with a concept that is still evolving. The classification distinguishes between three orthogonal aspects: what is the level of intelligence of the product, where is the intelligence (or processing power) located, and whether the product is managed as a single entity or as an aggregation. The reason for introducing such a threedimensional classification is that the earlier uni-dimensional classifications seemed to be under-developed either in the lower or the upper range of "intelligence" and did not necessarily take into account e.g. how the embedded processing capabilities affect the implementation of such Intelligent Products. Analyzing different approaches of Intelligent Products using the proposed three-dimensional classification makes it easier to identify the main limitations and aspects for improvement of a certain approach. However, the classification model proposed in this paper may still need to evolve as in the future more processing power, communication capabilities, sensors and actuators will be embedded into products.

The main technical foundations in the areas of automatic identification and embedded processing, distributed information storage and processing, and agent-based systems have been discussed. Regarding how, when and why to implement Intelligent Products, means-ends propositions for the Intelligent Product concept have been presented as a starting point for developing practical business cases in individual companies. This showed that the Intelligent Product can be employed to specific contexts such as manufacturing, supply chain, and asset management, as well as across contexts, i.e. for product life cycle management. In these contexts, globalization, virtual enterprises etc. will make it increasingly difficult to manage all information in centralized ways. Associating information, processing power and communication capabilities with products themselves and their surrounding environment can be an efficient way to relieve humans and corporate information systems from the management of routine operations. Therefore, we believe that the main contributions of Intelligent Products to manufacturing and supply chains are threefold. First of all, Intelligent Products enable their owners and users to know at any time the location and condition of their physical assets. Secondly, Intelligent Products can sense their location and condition and therefore can raise the red flag for "out-of-condition" situations. Thirdly, Intelligent Products allow to postpone decisions to the last moment, in such a way that adequate reaction to disturbances is possible.

Furthermore, we believe that Intelligent Products will have a visible impact on humans and society in the future. The decreasing price of embedded systems signifies that an increasing number of consumer products will become "intelligent". Through this evolution, we think new application domains will become attractive in addition to those mentioned in Section 4. Even though it is difficult to estimate how people appreciate new 
services enabled by Intelligent Products, we believe that they will be increasingly important e.g. for reducing energy consumption and for care of the elderly and disabled. The energy consumption of buildings, vehicles and machines, could be significantly reduced by better fault detection and control methods that would adapt to the state of the products and their environment. This increase in "intelligence" will also enable a better integration between infrastructure such as buildings, home electronics, mobile phones etc.

Monitoring the health of elderly people living in their homes will be facilitated by such technology, which might be the only way for many countries to take care of their aging population in a decent way and at a reasonable cost. It will also be interesting to see whether robotics will become important in the landscape of Intelligent Products. For the moment, it seems like humans would not be ready or interested in interacting with or being taken care of by human-like robots; however, people do accept autonomous grass-cutters, vacuum cleaners and other everyday devices that make their lives easier.

In order to make the above-mentioned scenarios possible, inter-operability and standardization plays an important role. There is still a long way to go before all different kind of machines and systems will be able to communicate and understand each other. Ontology- and semantic web-related research are expected to produce new tools for solving these inter-operability issues as no clear best solution exists yet. We also expect multi-agent systems and research to play an important role in the future.

\section{References}

[1] 3APL-M. Platform for lightweight deliberative agents. http://www.cs.uu.nl/3apl-m/.

[2] R.L. Albuquerque, J.F. Hubner, G.E. de Paula, J.S. Sichman, and G.L. Ramalho. Ksaci: A handheld device infrastructure for agents communication. In Pre-proc. of ATAL'01, pages 327-337, 2001.

[3] Rebecca Angeles. Rfid technologies: Supply-chain applications and implementation issues. Information Systems Management, 22(1):51-65, 2005.

[4] J. Anke and K. Främling. Distributed decision support in a plm scenario. In Proc. of Product Data Technology Europe 14th Symposium, 2005.

[5] Anonymous. Wal-mart expands rfid mandate. http://www.rfidjournal.com/, August 2003.

[6] Auto-ID-Labs. http://www.autoidlabs.org/.

[7] E. Bajic and F. Chaxel. Auto-id mobile information system for vehicle life cycle data management. In Proc. of 2002 IEEE SMC, 2002.

[8] C. Baumer, M. Breugst, S. Choy, and T. Magedanz. Grasshopper: a universal agent platform based on omg masif and fipa standards. In Proc. of MATA'99, pages 1-18, 1999.

[9] F. Bergenti and A. Poggi. Leap: A fipa platform for handheld and mobile devices. Lecture notes in computer science, 2333:436-446, 2002.

[10] Eric Bergman. Information appliances and beyond: interaction design for consumer products. Morgan Kaufmann, 2000.

[11] A. Brewer and T. Landers. Radio frequency identification: A survey and assessment of the technology. Technical report, University of Arkansas Department of Industrial Engineering, 1997.

[12] A. Brewer, N. Sloan, and T.L. Landers. Intelligent tracking in manufacturing. Journal of Intelligent Manufacturing, 10(3):245-250, 1999.

[13] David L. Brock. The electronic product code (EPC)-a naming scheme. Technical Report MIT-AUTOIDWH-002, 2001.

[14] S. Bussmann and D. McFarlane. Rationales for holonic manufacturing systems. In Proc. of Workshop on IMS'99, pages 177-184, 1999. 
[15] S. Bussmann and K. Schild. Self-organizing manufacturing control: An industrial application of agent technology. In Proc. of the MAS'00, pages 10-12, 2000.

[16] C. Carabelea and O. Boissier. Multi-agent platforms on smart devices: Dream or reality? In Proc. of SOC'03, pages 126-129, 2003.

[17] C. Carabelea, O. Boissier, and F. Ramparany. Benefits and requirements of using multi-agent systems on smart devices. LNCS, 2790:1091-1098, 2004.

[18] J. Cassina, M. Tomasella, M. Marquard, A. Metin, A. Matta, and M. Taisch. Development of the semantic object model for a pdkm system. In Proc. of ICE 2006, 2006.

[19] Jonathan Collins. Suppliers must 'slap and ship'. http://www.rfidjournal.com/, April 2004.

[20] Cecile Corcelle, Kary Främling, Lutz Rabe, Jürgen Anke, and Jouni Petrow. Assessment of itemspecific information management approaches in the area of heavy load vehicles. In Proceedings of the International Conference on Product Lifecycle Management (PLM'07), pages 773-782, 2007.

[21] A. Davie. Intelligent tagging for transport and logistics: the parcelcall approach. Electronics 63 Communication Engineering Journal, 14(3):122-128, 2002.

[22] Dialog. http://dialog.hut.fi/.

[23] e INSIDER. The role of rfid in b2b trade. http://www.supplychainbrain.com/news/e12.04.02.newsletter.htm, 2002.

[24] EPC-Global. http://www.epcglobalinc.org/.

[25] T. Feare. Pump up the volume. Modern Materials Handling, 55(3):55-59, 2000.

[26] K. Främling, J. Holmström, T. Ala-Risku, and M. Kärkkäinen. Product agents for handling information about physical objects. Technical report, Helsinki University of Technology, 2003.

[27] Kary Främling, Timo Ala-Risku, Mikko Kärkkäinen, and Jan Holmström. Design patterns for managing product life cycle information. Communications of the ACM, 50(6):75-79, 2007.

[28] Kary Främling, Mark Harrison, James Brusey, and Jouni Petrow. Requirements on unique identifiers for managing product lifecycle information - comparison of alternative approaches. International Journal of Computer Integrated Manufacturing, 20(7):715-726, 2007.

[29] Kary Främling and Jan Holmström. Design patterns for managing product lifecycle information. In Proceedings of 18th International Conference on Production Research (ICPR), 31 July to 4 August 2005, Salerno, Italy, 2005.

[30] Kary Främling, Mikko Kärkkäinen, Timo Ala-Risku, and Jan Holmström. Agent-based model for managing composite product information. Computers in Industry, 57(1):72-81, 2006.

[31] J.W. Gardner and O.O. Awadelkarim. Microsensors, MEMS, and Smart Devices. John Wiley and Sons, 2001.

[32] Hans W. Gellersen, Albrecht Schmidt, and Michael Beigl. Multi-sensor context-awareness in mobile devices and smart artifacts. Mobile Networks and Applications, 7(5):341-351, 2004.

[33] N. Gershenfeld, R. Krikorian, and D. Cohen. The internet of things. Scientific American, 291(4):76-81, 2004.

[34] Neil Gershenfeld. When Things Start to Think. Owl books, 2000.

[35] J.E. Hennig, P.B. Ladkin, and B. Sieker. Privacy enhancing technology concepts for rfid technology scrutinised. Technical report, Research Report RVS-RR-04-02, University of Bielefeld, D-33501 Bielefeld, Germany, 2004. 
[36] E. Huvio, J. Grönvall, and K. Främling. Tracking and tracing parcels using a distributed computing approach. In Proc of NOFOMA'02, pages 29-43, 2002.

[37] B. Ives and M.R. Vitale. After the sale: Leveraging maintenance with information technology. MIS Quarterly, 12(1):7-21, 1988.

[38] JADE. http://jade.cselt.it/.

[39] Nicholas R. Jennings and Michael J. Wooldridge. Applications of intelligent agents. In Nicholas R. Jennings and Michael J. Wooldridge, editors, Agent Technology: Foundations, Applications, and Markets, pages 3-28. Springer-Verlag: Heidelberg, Germany, 1998.

[40] NR Jennings, JM Corera, and I. Laresgoiti. Developing industrial multi-agent systems. In Proc. of the First International Conference on Multi-agent Systems, pages 423-430, 1995.

[41] D. Johnson. Rfid tags improve tracking, quality on ford line in mexico. Control Engineering, 49(11):16$17,2002$.

[42] P. Jones, C. Clarke-Hill, D. Hillier, and D. Comfort. The benefits, challenges and impacts of radio frequency identification technology (rfid) for retailers in the uk. Marketing Intelligence $\& 3$ Planning, (4):395-402, 2005.

[43] Omar Kahn, Andrea Scotti, Alessandro Leverano, Francesco Bonini, Giorgio Ruggiero, and Christian Dörsch. Rfid in automotive: a closed-loop approach. In Proc. of ICE'06, 2006.

[44] R. S. Kaplan. Innovation action research: Creating new management theory and practice. Journal of Management Accounting Research, 10:89-118, 1998.

[45] D. Kiritsis. Ubiquitous product lifecycle management using product embedded information devices. In Proc. of IMIS'04, 2004.

[46] F. Koch, J.J.C. Meyer, F. Dignum, and I. Rahwan. Programming deliberative agents for mobile services: the 3apl-m platform. LNAI, 3862:222-235, 2006.

[47] M. Kärkkäinen, J. Holmström, K. Främling, and K. Artto. Intelligent products - a step towards a more effective project delivery chain. Computers in Industry, 50:141-151, 2003.

[48] Mikko Kärkkäinen, Timo Ala-Risku, and Kary Främling. Efficient tracking for short-term multi-company networks. Int. J. of Physical Distribution and Logistics Management, 34(7):545-564, 2004.

[49] Mikko Kärkkäinen, Timo Ala-Risku, Kary Främling, and Jari Collin. Establishing inventory transparency to temporary storage locations. In Proceedings of Advances in Production Management Systems (APMS), 18-21 September 2005, Washington, USA, 2005.

[50] Marc Langheinrich. Privacy by design - principles of privacy-aware ubiquitous systems. LNCS, 2201:273$291,2001$.

[51] Marc Langheinrich. Rfid and privacy. In Milan Petkovic and Willem Jonker, editors, Security, Privacy, and Trust in Modern Data Management, pages 433-450, Berlin, 2007. Springer.

[52] J.H. Lee and C.O. Kim. Multi-agent systems applications in manufacturing systems and supply chain management: a review paper. International Journal of Production Research, 46(1):233-265, 2008.

[53] S. Ljungblad, T. Skog, and L. Gaye. Are designers ready for ubiquitous computing?: a formative study. In Proc. of CHI'03, pages 992-993, 2003.

[54] Q.H. Mahmoud. Mobiagent: An agent architecture and platform for wireless information systems. In Proc. of AOIS'01, 2001.

[55] D. McFarlane, S. Sarma, J.L. Chirn, CY Wong, and K. Ashton. Auto id systems and intelligent manufacturing control. Engineering Applications of Artificial Intelligence, 16(4):365-376, 2003. 
[56] Micro-FIPA-OS. http://fipa-os.sourceforge.net/index.htm.

[57] T. Minami. Needs and benefits of massively multi book agent systems for u-libraries. Lecture Notes in Artificial Intelligence, 3346:239-253, 2005.

[58] I. Niiniluoto. The aim and structure of applied research. Erkenntnis, 38:1-21, 1993.

[59] P. Nikulainen. Final report: Jobsite logistics (mobile rfid). Technical report, TeliaSonera, Helsinki, 2005.

[60] Donald Arthur Norman. The invisible computer: why good products can fail, the personal computer is so complex, and information appliances are the solution. MIT Press, 1998.

[61] K. R. Popper. Conjectures and Refutations. Routledge, London, 1963.

[62] PROMISE. http://www.promise.no/.

[63] F. Ramparany and O. Boissier. Smart devices embedding multi-agent technologies for a pro-active world. In Proc. of the Ubiquitous Computing Workshop, 2002.

[64] SACI. Simple agent communication infrastructure. http://www.lti.pcs.usp.br/saci/.

[65] Sanjay Sarma, David L. Brock, and Kevin Ashton. The networked physical world. Technical Report MIT-AUTOID-WH-001, October 2000.

[66] W. Shen, Q. Hao, H.J. Yoon, and D.H. Norrie. Applications of agent-based systems in intelligent manufacturing: An updated review. Advanced Engineering Informatics, 20:415-431, 2006.

[67] F. Siegemund and C. Florkemeier. Interaction in pervasive computing settings using bluetooth-enabled active tags and passive rfid technology together with mobile phones. In Proc. of PerCom'03, pages 378-387, 2003.

[68] Herbert A. Simon. The sciences of the artificial (3rd ed.). MIT Press, Cambridge, MA, USA, 1996.

[69] Smart-Its. http://www.smart-its.org/.

[70] B. Srivastava. Radio frequency id technology: The next revolution in scm. Business Horizons, 47(6):60$68,2004$.

[71] Frank Stajano. Security for Ubiquitous Computing. John Wiley \& Sons, Chichester, UK, 2002.

[72] William Stone, Alan Lytle, and Karen Furlani. Smart chips in construction. Technical report, National Institute of Standards and Technology, 2002.

[73] M. Strassner and T. Schoch. Todays impact of ubiquitous computing on business processes. In Proc. of Pervasive'02, pages 62-74, 2002.

[74] ToolWatch. http://www.toolwatch.com/.

[75] Trackway. Trackway. http://www.trackway.eu/.

[76] TraSer. http://www.traser-project.eu/.

[77] S. Coble Trovinger and R. Bohn. Setup time reduction for electronics assembly: Combining simple (smed) and it-based methods. Production and Operations Management, 14(2):205-217, 2005.

[78] J. E. van Aken. Management research based on the paradigm of design sciences: The quest for field-tested and grounded technological rules. Journal of Management Studies, 41:219-246, 2004.

[79] Olli Ventä. Intelligent products and systems. Technical report, VTT, 2007.

[80] Mark Weiser. The computer for the twenty-lirst century. Scientific American, 265(3):94-104, September 1991. 
[81] Mark Weiser. Some computer science issues in ubiquitous computing. Commun. ACM, 36(7):75-84, July 1993.

[82] C.Y. Wong, D. McFarlane, A. Zaharudin, and V. Agarwal. The intelligent product driven supply chain. In Proc. of SMC'02, 2002.

[83] WWAI. World wide article information. http://www.wwai.org/.

[84] L. Zhekun, R. Gadh, and BS Prabhu. Applications of rfid technology and smart parts in manufacturing. In Proc of DETC'04, 2004. 